ministers since the infamous late Dr Manto Tshabalala-Msimang has slowly rebuilt confidence in it.

The trial team observed no substantive safety concerns from use of the gel and no increase in risky behaviour by the women.

Karim said her team's next step was to understand why 38 women in the tenofovi arm of their double-blind study contracted HIV infections. The study participants had 'almost uniformly' asked her when this study of the gel would take place and expressed their availability to participate. They were very keen to know when the product would be available for general use.

The CAPRISA 004 trial saw 98 of the 889 participants become HIV positive - 38 out of 445 in the tenofovir gel group and 60 out of 444 in the placebo gel group. Out of the 434 women who tested negative for genital herpes at the start of the trial, 29 became infected in the tenofovir group and 58 in the placebo group. The women were advised to use the gel up to 12 hours before sex and soon after having sex for a maximum of 2 doses in 24 hours - a dosing strategy referred to as BAT24. Participants used the gel for a minimum of 1 year and a maximum of 2.5 years. The trial team observed no substantive safety concerns from use of the gel and no increase in risky behaviour by the women.

\section{Researchers 'stunned' by study outcome}

Professor Salim Abdool Karim, Pro ViceChancellor at the University of KwaZuluNatal and CAPRISA Director, said he was stunned when the statistician came in and gave us the results. We just looked at each other in disbelief and couldn't speak for a while.' The couple and their team's work has been solidly grounded in population-based studies, with the alarming age and gender graph showing a sharp rise in infections for women from 15 years old versus men,

'This has the potential to alter the course of the HIV epidemic, especially in southern Africa where young women bear the brunt of this devastating disease.' for whom this trend occurs 8 years later. A full $60 \%$ of all new infections occur among women and girls.

'As the epidemic matured we stayed focused on things that would have the biggest public health impact and rooted our work in what the epidemiological data told us,' Dr Abdool Karim said. Her husband added, 'This has the potential to alter the course of the HIV epidemic, especially in southern Africa where young women bear the brunt of this devastating disease.' A spokesperson for the TIA said her agency's role in product development was to ensure the MCC received all the information required to make a decision 'as soon as possible'. Once approved for use in South Africa, the TIA was responsible for ensuring that the tenofovir gel was manufactured and distributed affordably in Africa. 'We're committed to ensure women in Africa get access to the product a soon as it is approved by the regulatory authorities,' she added.

Chris Bateman

\title{
Bullish new medical measures to give more pilots wings
}

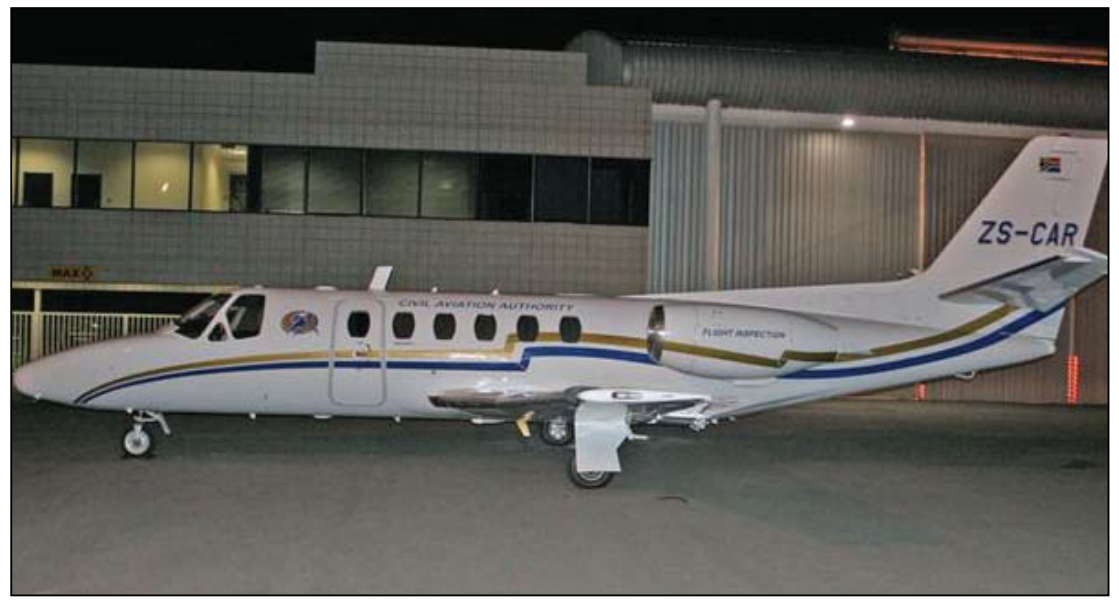

The Civil Aviation Authority (CAA) plane used for crashed investigations.
Pilots, cabin crew members and air traffic controllers, frustrated by outdated protocols for medical certification which lead to lengthy delays and unnecessary and expensive appeals, will by this September begin benefiting from a major systemic upgrade.

An over-reliance by South Africa's Civil Aviation Authority (CAA) on the underresourced military for the verification of medical certificates plus the lack of a clear definition of 'acceptable medical risk' are now being addressed as top priorities.

A vital additional quality assurance instrument will be the newly formed 15-member Aeromedical Committee, established by the CAA late this April to 
supplement and bolster the SA Military Health Services' longstanding Institute for Aviation Medicine (IAM).

Explaining the reasons for the overhaul, Dr Lesego Bogatsu, Senior Manager for Aviation Medicine at the CAA, said insufficient specialists available to adjudicate cases plus the redeployment of military doctors meant verification backlogs had built up.

\section{Bogatsu's baseline research} shows that $97 \%$ of all applicants who appealed their medical grounding via the civil aviation commissioner between January 2000 and December 2008 were later found to be medically fit by an independent panel of specialist advisors.

Other challenges included outdated medical protocols (some of them requiring identical medical criteria for cabin crew members, pilots and air traffic controllers), the lack of an integrated information technology system between stakeholders, administrative errors and limited participation of medical universities in the overall risk minimising process.

She said that Colonel Noel Ndlhovu, Officer in Command at the IAM, had 'given his word' that the verification backlog would be cleared within 3 months.

\section{Most appeals succeeded}

Bogatsu's baseline research shows that $97 \%$ of all applicants who appealed their medical grounding via the civil aviation commissioner between January 2000 and December 2008 were later found to be medically fit by an independent panel of specialist advisors.

She discovered that there was 'limited understanding' of and confusion in the interpretation of the Civil Aviation Technical Standards, a typical example being the finding of applicants 'permanently unfit', a category not included in the standards.

The terms 'temporarily unfit' and 'unfit' in defining medical standards were used interchangeably, leading to confusion among applicants, unnecessary medical appeals and even litigation.
Her investigation which forms part of her Master's thesis, plus advances in medical science and technology, required the CAA to 'urgently reduce the number of medical unfitness appeals' by (among other things) investing in research.

There was a need to define acceptable medical risk to suit the South African environment and to implement strategies to mitigate the risk to the CAA, while terms such as 'medical unfitness', 'temporary medical unfitness' and 'unfitness' needed clearer definition to reduce medical appeals and litigation. Another shortfall was the lack of routine collection and analysis of data on incapacitation in-flight and on active duty.

South Africa was one of the few countries that strongly disadvantaged licensed cabin crew and recreational pilots by requiring the higher medical protocols applicable to commercial pilots.

\section{Comprehensive review}

Bogatsu reviewed 2521 cases presented at the Institute for Aviation Medicine over 8 years (Jan 2000 - Dec 2008). She found that patterns of morbidity most commonly associated with temporary unfitness, unfitness and 'permanent unfitness' were neurological, cardiovascular and psychiatric.

\section{He said the IAM and the CAA} had agreed to actively recruit appropriately qualified doctors

to help clear the verification backlog and provide additional training to improve the quality assurance skills needed. Gaps in the medical protocols had been identified and outdated protocols would be reviewed by the end of September this year.

Most disqualifications were related to the central nervous system (29.34\%), with head injuries and convulsions the most common in this medical category. The cardiovascular system accounted for the second most common cause of medical unfitness, with coronary artery disease and hypertension dominant. Psychiatric conditions (12.9\%) were the third most common, with depression

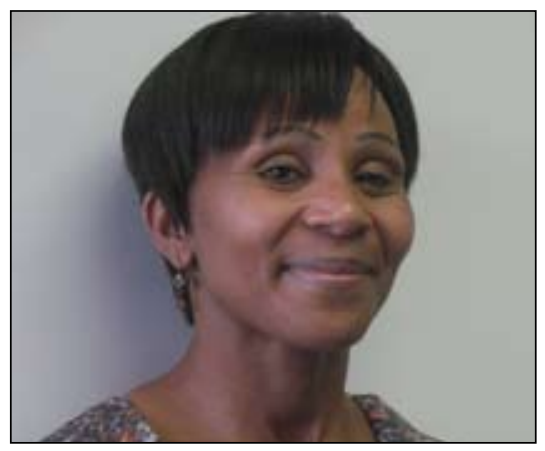

Dr Lesego Bogatsu, Senior Manager for Aviation Medicine at the Civil Aviation Authority (CAA).

and substance abuse responsible for most medical groundings in this discipline.

Captain Colin Jordaan, Director of the CAA, said at the launch of the new Aeromedical Committee at the Rand Airport on 28 April that its composition of experts from various medical universities and organisations would ensure accredited medical conclusions were reached and that the CAA kept abreast with International Best Practice.

He said the IAM and the CAA had agreed to actively recruit appropriately qualified doctors to help clear the verification backlog and provide additional training to improve the quality assurance skills needed. Gaps in the medical protocols had been identified and outdated protocols would be reviewed by the end of September this year.

Captain Jordaan said that 'the human component' was the cause of $70 \%$ of aviation accidents. The aircraft environment differed from other occupational environments in respect of altitude stressors. These included hypoxia, noise and vibration, low humidity (leading to dehydration), fatigue, decompression syndrome, acceleration and spatial disorientation.

Aviation medicine came into existence during World War I, when it was realised that more pilots died due to medical incapacity than were killed by enemy gunfire. Once medical standards were introduced, fatality rates dropped 'significantly'.

\section{Chris Bateman}

